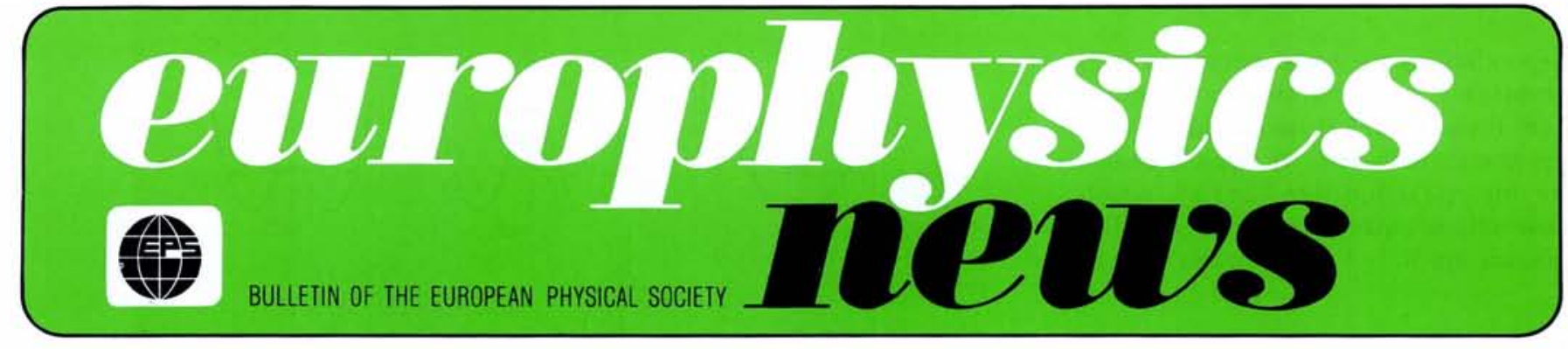

J.A.

\title{
Dielectric Spectroscopy of Soft Modes in Ferroelectrics
}

\section{J. Petzelt, Prague,}

(Institute of Physics, Czechoslovak Academy of Sciences)

\section{G.V. Kozlov and A.A. Volkov, Moscow}

\author{
(Institute of General Physics, USSR Adademy of Sciences)
}

The aim of dielectric spectroscopy is to determine the complex dielectric function $\varepsilon(\omega)=\varepsilon^{\prime}(\omega)-i \varepsilon^{\prime \prime}(\omega)$ as a function of frequency $\omega$. In a normal dielectric, the main contribution to the infrared spectrum $10-10^{4} \mathrm{~cm}^{-1}(1000-1 \mu \mathrm{m})$ originates from polar lattice vibrations and the dielectric dispersion is, as a rule, of the resonance (underdamped oscillator) type. At lower frequencies no dielectric dispersion is expected in the case of an ideal harmonic crystal, except for piezoelectric resonances in non-centrosymmetric crystals. In strongly anharmonic and partially disordered crystals, however, additional dielectric dispersion can occur in the microwave range $\left(10^{-1}\right.$. $10 \mathrm{~cm}^{-1}$ ), or even lower, which is connected with relatively large - amplitude fluctuations in the positions of disordered particles. This dispersion is, as a rule, of the relaxational loverdamped, diffusive) type. In such systems the greatest attention has been paid to crystals exhibiting structural phase transitions out of which the most important class is the ferroelectric. The increase of interest in such systems during the last 20 years is connected with new physical phenomena occurring near phase transitions.

Soft Modes in Displacive and Orderdisorder Systems

The existence of soft modes represents the central concept of all dynamical theories of structural phase transitions. In general terms, a soft mode is a collective excitation whose energy tends to zero when the temperature approaches the critical temperature $T_{c}$. This causes an instability of the crystal against fluctuations of the soft-mode displacements. The soft mode beha- viour is of primary importance for structural phase transitions because it determines a number of anomalies in properties near $T_{c}$ through its coupling with other excitations. Two extreme categories of structural phase transition differing by the character of the soft mode displacements can be distinguished:

\section{(a) Displacive Phase Transitions}

In this case the soft mode is one of the normal vibrational modes (phonon) and the structural change below $T_{c}$ consists of a freezing of the soft-mode eigenvector. If the soft-mode is dielectrically active, i.e. if its eigenvector has a non-zero dipole moment (which is always the case for ferroelectric phase transitions), its contribution to the dielectric spectrum is expected to be that of a classical oscillator (see Fig. 1a):

$$
\Delta \varepsilon_{\mathrm{s}}(\omega)=\frac{f}{\omega_{\mathrm{s}}^{2}(T)-\omega^{2}+\mathrm{i} \omega \Gamma}
$$

where $f$ is the oscillator strength. If mean field theory is valid, the soft-mode eigenfrequency varies like $\omega_{s}(T) \propto$ $\left(T_{\mathrm{c}}\right)^{1 / 2}$ near $T_{\mathrm{c}}$. Typically, $\omega_{\mathrm{s}}$ lies in the far-infrared region $\left(10-100 \mathrm{~cm}^{-1}\right)$ not too close to $T_{c}$. The damping constant should be frequency-independent and in the first approximation temperature-independent as well. Typically $\Gamma \sim 10-50$ $\mathrm{cm}^{-1}$, so that far from $T_{\mathrm{c}}$, the soft mode is underdamped $\left(\Gamma<2 \omega_{\mathrm{s}}\right)$, but close to $T_{\mathrm{c}}$, it becomes overdamped $\left(\Gamma>2 \omega_{\mathrm{s}}\right)$.

\section{(b) Order-disorder Phase Transitions}

In this case the soft mode is not one of the normal lattice modes but rather an additional excitation which describes the coherent hopping of the disordered particles among various allowed sites. The freezing of the soft-mode displace- ments means in this case a collective ordering of the particles. If quantum tunnelling can be neglected (which is nearly always the case), the soft-mode response is expected to be overdamped at all temperatures. If, moreover, the time which a particle spends between sites can be neglected with respect to its dwell time about a given site, a pseudospin model can be used which ascribes a discrete variable to each site. Then one can show the contribution to the dielectric spectrum (see Fig. 1b) to be of the Debye relaxation type:

$\Delta \varepsilon_{\mathrm{s}}(\omega)=f^{\prime} \lambda \tau_{\mathrm{s}}(T) /\left\{1+\mathrm{i} \omega \tau_{\mathrm{s}}(T)\right\}$.

Here $f^{\prime}$ is the relaxation strength which is determined by the effective dipole moment of the pseudospin; $\lambda$ is a kinetic coefficient in the corresponding basic equation of irreversible thermodynamics which is expected to be roughly independent of temperature; $\tau_{\mathrm{s}}(T)$ is a temperature-dependent relaxation time.

In mean field theory $\tau_{\mathrm{s}}^{-1}(T) \propto\left(T-T_{c}\right)$, which again yields a Curie-Weiss law for $\Delta \varepsilon_{\mathrm{s}}(0)$ if $f^{\prime}$ is temperature-independent. It should be noted that the mean-field

\section{Contents}

Dielectric Spectroscopy of Soft Modes in Ferroelectrics

New Members of EPS 1

Experiments at LEAR

Fundamental Constants \& SI Units 8

Biomagnetism

EPS History

Delegates of Associate Members 15

CMD Liquids Section 16 
dependences are not expected to hold close to $T_{c^{\prime}}$, but we shall not discuss here these so-called non-classical critical phenomena (Ref. 1). Typical values for the relaxation rate $\left(2 \pi \tau_{\mathrm{s}}\right)^{-1}$, which now characterizes the soft-mode frequency, are $10^{-1}-1 \mathrm{~cm}^{-1}$, not too close to $T_{\mathrm{c}}$.

\section{History}

The history of our subject began in the early sixties shortly after the theoretical prediction of soft modes in displacive ferroelectrics independently by $W$. Cochran, P. Anderson and V.L. Ginzburg. In 1962, soft-mode behaviour was first observed by A.S. Barker, Jr. and $M$. Tinkham in the far infrared reflectivity of the incipient displacive ferroelectric $\mathrm{SrTiO}_{3}$. In the same year, critical slowing-down of the relaxation rate was found by R.M. Hill and S.K. Ichiki in the order-disorder ferroelectric triglycine sulphate (TGS) using microwave coaxial-line and waveguide techniques up to $4 \times 10^{10} \mathrm{~Hz}\left(1.3 \mathrm{~cm}^{-1}\right)$. Afterwards, several soft modes were observed in the dielectric spectra of various ferroelectrics (see Refs. 2-5), but the experimental accuracy, especially above $\sim 1 \mathrm{~cm}^{-1}$ was not high enough to study the softmode behaviour on a satisfactory quantitative level. In the meantime, inelastic light and neutron scattering techniques developed rapidly, and the number of publications devoted to soft-mode spectroscopy using these techniques considerably exceeded that of similar publications based on far-infrared or microwave techniques. However, during the last ten years new infrared techniques have been developed and renewed interest and activity in the dielectric spectroscopy of soft modes can be noted.

\section{Modern Infrared Techniques}

The most interesting results are currently being obtained using two techniques: (i) rapid-scan Fourier transform spectroscopy and (ii) backward wave oscillator (BWO) spectroscopy. The former technique is now commercially available for the entire infrared range (10 $-10^{4} \mathrm{~cm}^{-1}$ ). It relies on normal bulk reflectivity measurements which are subsequently evaluated by means of phenomenological fitting procedures or Kramers-Kronig analysis to obtain the dielectric spectrum. It has been pursued mainly at the Institute of High Temperature Physics in Orléans.

The latter technique, which works in the difficult spectral range $3-30 \mathrm{~cm}^{-1}$, especially appropriate for soft-mode studies in ferroelectrics, has been developed mainly at the Institute of General Physics in Moscow, and is not yet com- a
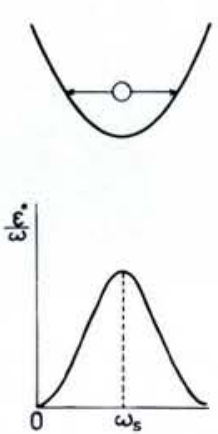

b
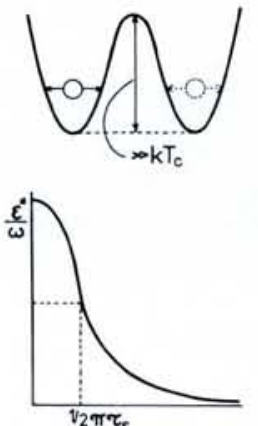

c
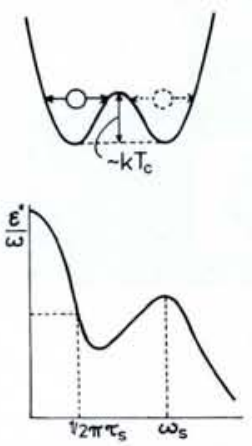

Fig. 1 - Schematic views of soft-mode particle motion in effective mean-field potential corresponding spectra of $\varepsilon^{\prime \prime}(\omega) / \omega$ (proportional to the dynamic structure factor). a: displacive limit, b: order-disorder limit, $c$ : intermediate (crossover) case.

mercially available. It is based on monochromatic sources, backward-wave oscillators, which are broadly tunable by an applied voltage. The quality of the radiation (power 1 - $10 \mathrm{~mW}$, monochromaticity $v / \Delta v \sim 10^{5}$ and degree of polarization $99.99 \%$ ) is similar to that found in microwave techniques, but otherwise the technique is based on simple optical principles: independent measurements of the power and phase shift of a wave transmitted through a plane-parallel sample. On-line connection with a controlling minicomputer enables a real time evaluation of dielectric spectra to be made. Varying the sample thickness makes possible a precise determination of dielectric spectra over a broad range $\left(\varepsilon^{\prime} \sim-10^{3} \div 10^{3}, \varepsilon^{\prime \prime} \sim 10^{-3} \div 10^{3}\right)$. Unlike reflectivity measurements, this method is independent of any model for dielectric dispersion and no fitting procedure is needed to obtain the dielectric spectra.

\section{Main Results}

So far more than 30 ferroelectric crystals have been investigated in the vicinity of their phase transitions using the described BWO technique. Some of the striking results are discussed below.

\section{Displacive Ferroelectrics}

a) In some crystals which were believed to be of the displacive type, a crossover between displacive and order-disorder behaviour has been observed near $T_{\text {c }}$. The soft-mode response in such crystals follows the displacive behaviour far away from $T_{c}$. However, near $T_{c}$ an additional relaxation peak arises in the spectrum which softens at $T_{c^{\prime}}$ whereas the soft mode saturates at a finite frequency for $T \rightarrow T_{c}$. The additional relaxation also produces an increasing central peak in the inelastic scattering spectrum near $T_{c}$ which has been observed in phase-transition studies using Raman and neutron scattering. However, the half-width of the central peak, which determines the relaxation frequency $\left(2 \pi \tau_{\mathrm{s}}\right)^{-1}$, could seldom be resolved by those techniques; moreover, such central peaks seem to be mostly of an extrinsic (defect) nature. On the other hand, dielectric spectroscopy clearly shows the existence of an additional intrinsic (not too much sample dependent) relaxation near $T_{\mathrm{c}}$ in the $1-20 \mathrm{~cm}^{-1}$ region in displacive ferroelectrics like $\mathrm{BaTiO}_{3}, \mathrm{KNbO}_{3}$ (see Ref. 6), SbSI or $\mathrm{Gd}_{2}\left(\mathrm{MoO}_{4}\right)_{3}$. The most probable explanation of this behaviour is a partial disorder in the crystals near $T_{c}$. In simple terms this means that the particles involved in the soft mode with wave vectors close to that of soft-mode move near $T_{c}$ in a double (or multi)-minimum potential with a potential barrier between minima of the order of the thermal energy $k T_{c}$ (the distance between mini$\mathrm{ma}$ is of the order of $0.1 \AA$ ). The associated soft-mode spectrum consists of at least two peaks (see Fig. 1c), the higher-frequency corresponding to the small-amplitude oscillations in a single minimum (classical soft mode)

Fig. 2 - Soft mode spectra of GMO and fits to a simple phenomenological coupled-mode model. $T_{c}=432 \mathrm{~K}$. (After Petzelt et al., to be publ.)

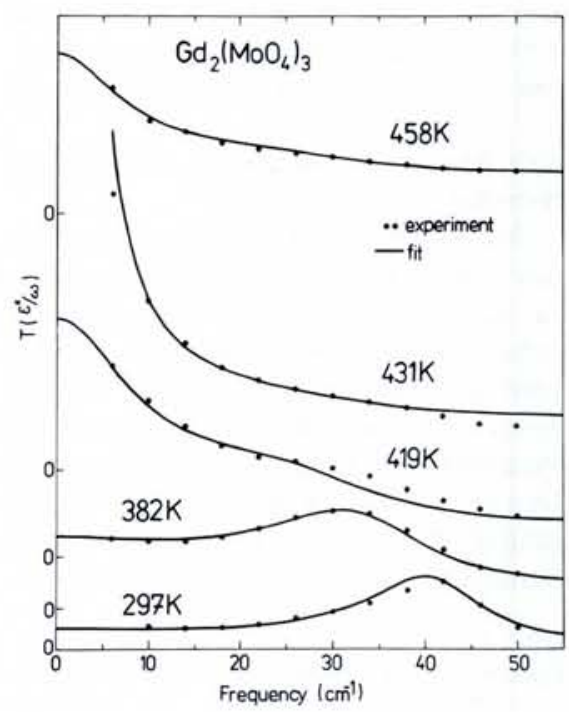




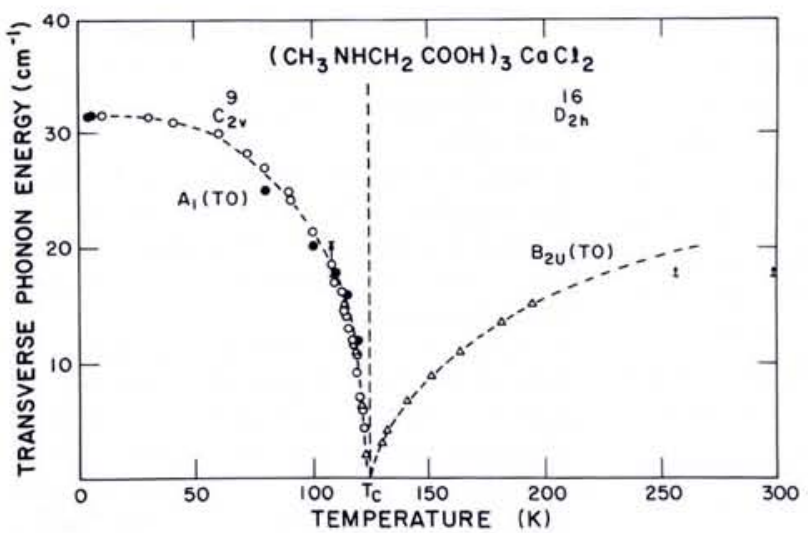

Fig. 3 - Temperature dependence of the soft mode frequency in TSCC. The triangles are from BWO data, the circles are from Raman measurements. (After Ref. 8).

and the lower-frequency to the largeamplitude hopping among different minima (central mode). The first model calculation exhibiting this behaviour was performed by Y. Onodera in 1970 and has since then been confirmed by more refined calculations and computer simulations reviewed in Ref. 1.

Significant progress was recently achieved in the case of the improper ferroelectric $\mathrm{Gd}_{2}\left(\mathrm{MoO}_{4}\right)_{3}(\mathrm{GMO})$ where the complete dielectric spectrum in the 5 $50 \mathrm{~cm}^{-1}$ region was determined in detail and fitted to a phenomenological theory based on a coupled-mode formalism. In improper ferroelectrics, the polarization is not the order parameter and, as a consequence, the soft mode is dielectrically inactive above $T_{c}$; it becomes weakly active below $T_{c}$ only because of an interaction of the soft mode with other polar modes (see Ref. 7). The resulting experimental data together with the fitting spectra are shown in Fig. 2. To demonstrate the growth of the central peak, $T \varepsilon^{\prime \prime}(\omega) / \omega$ is plotted as this quantity is proportional to the dynamical structure factor measured in inelastic scattering experiments. The most striking feature (resulting from the parameters of the fit) is the nonlinear increase of the coupling between the soft and central modes near $T_{c}$.

b) In some other displacive ferroelectrics, no intrinsic central mode appears. This has been nicely demonstrated on the molecular ferroelectric trissarcosine calcium chloride $\mathrm{CH}_{3} \mathrm{NHCH}_{2}$ $\mathrm{COOH})_{3} \mathrm{CaCl}_{2}$ (TSCC). Several recent theoretical approaches have assumed an order-disorder mechanism for the phase transition at $T_{c} \sim 130 \mathrm{~K}$. However BWO measurements in the $3-18 \mathrm{~cm}^{-1}$ region revealed an underdamped softmode both above and below $T_{c}$ shown in Fig. 3. It accounts for the whole static dielectric anomaly; TSCC is a textbook example of a classical displacive system.

\section{Hydrogen-bonded Ferroelectrics}

Very recently, a new understanding of the proton dynamics in classical hydro- gen-bonded $\mathrm{KH}_{2} \mathrm{PO}_{4}$ (KDP) has grown up. After 20 years of belief that quantum tunnelling of protons in a double-well potential is the driving mechanism for the ferroelectric phase transition, it has become clear that the situation is rather more prosaic: The protons are strongly coupled to $\mathrm{PO}_{4}$ tetrahedraforming $\left(\mathrm{H}_{2} \mathrm{PO}_{4}\right)^{-}$ions which perform classical hopping motion between two configurations. There is now much structural as well as Raman and infrared spectroscopic evidence for such a picture, including the purely relaxational softmode behaviour evident from BWO spectroscopy. In Fig. 4 the temperature dependence of the soft-relaxation frequency is shown for the mixed system $\mathrm{KH}_{2(1-x)} \mathrm{D}_{2 \mathrm{x}} \mathrm{PO}_{4}$ with different deuterium concentrations. The single relaxation behaviour observed in the whole composition range points to the importance of long-range forces. The results need a theoretical reinterpretation in the light of the phase-transition mechanism mentioned above.

\section{Order-disorder Ferroelectrics}

Rochelle salt (RS), $\mathrm{NaKC}_{4} \mathrm{H}_{4} \mathrm{O}_{6} \cdot 4 \mathrm{H}_{2} \mathrm{O}$ was the first ferroelectric, discovered in 1921 by J. Valasek. However, its properties are by no means simple and remained incompletely understood until now. It exhibits two phase transitions at $255 \mathrm{~K}$ and $297 \mathrm{~K}$ and only the intermediate phase is ferroelectric. The structure below $255 \mathrm{~K}$ and above $297 \mathrm{~K}$ is nonpolar (but piezoelectric) and identical with respect to symmetry. Both transitions

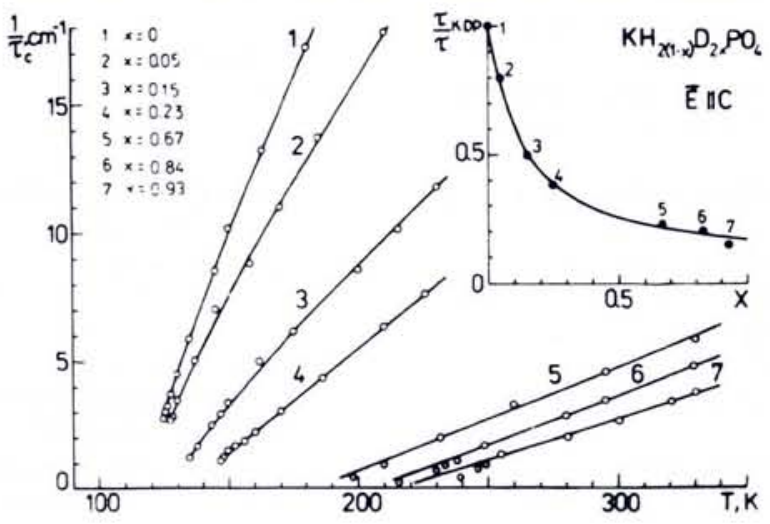

Fig.4 - Soft relaxation frequencies in the

$\mathrm{KH}_{2(1-x)} \mathrm{D}_{2 x} \mathrm{PO}_{4}$ system as function of temperature and deuteration. The inset shows the ratio of the soft mode frequencies of partially deuterated crystals to that in KDP for a fixed temperature interval $T-T_{c}$. (After Ref. 9). are second order and the soft mode is relaxational.

Recent BWO measurements have shown that its temperature dependence between 180 and $250 \mathrm{~K}$ follows a rather unusual cubic law

$$
\left(2 \pi \tau_{\mathrm{s}}\right)^{-1}=1.07\left(T_{0}-T\right)^{3} \times 10^{-5} \mathrm{~cm}^{-1}
$$
with $T_{0}=275 \mathrm{~K}$. A similar law was also found in the deuterated salt DRS:

$\left(2 \pi \tau_{\mathrm{s}}\right)^{-1}=0.8\left(T_{0}-T\right)^{3} \times 10^{-5} \mathrm{~cm}^{-1}$ with $T_{0}=280 \mathrm{~K}$. The difference between $\stackrel{\circ}{\circ}_{0}$ and the actual transition temperature is caused by a piezoelectric coupling between the soft mode and a corresponding acoustic mode. The cubic law as well as the existence of two transitions can now be understood in the framework of a model developed by Mitsui already in 1958 which assumes two asymmetric double-minimum potentials for the disordered particles in the unit cell (Ref. 10).

\section{Incommensurate Ferroelectrics}

Incommensurate ferroelectrics are characterized by an incommensurate phase which precedes the ferroelectric phase upon cooling the crystal from the normal nonpolar phase. In this intermediate phase the structure exhibits a frozen-in polarization wave of a period incommensurate with and much larger than the lattice spacing (see Ref. 11). This period grows upon approaching the ferroelectric transition and the polarization wave changes into a periodic ferroelectric domain structure close to the ferroelectric transition. From the point of view of lattice dynamics the incommensurate phase is characterized by new low-frequency vibrations, namely by fluctuations of the amplitude and phase of the incommensurate modulation wave, so-called amplitudons and phasons. Both branches originate from the soft branch in the normal phase; the distinguishing feature of an ideal incommensurate phase is that the phason branch tends linearly to zero with the wave vector $\mathbf{k} \rightarrow 0$.

The behaviour of amplitudons is similar to that of the soft mode below a usual displacive phase transition and in conse- 


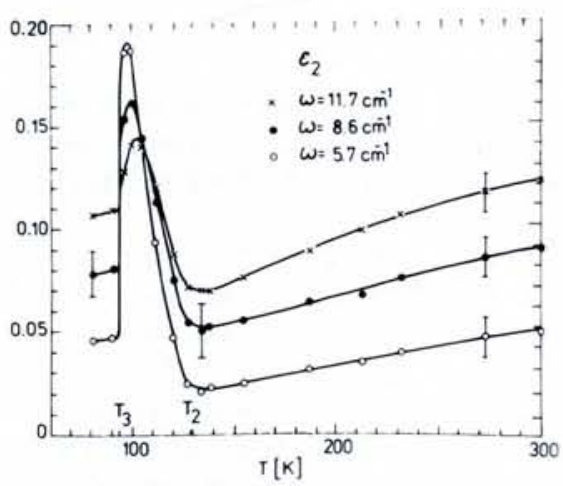

Fig. 5 - Temperature dependence of dielectric losses in $\mathrm{K}_{2} \mathrm{SeO}_{4}$. The structure between $T_{2}$ and $T_{3}$ is incommensurate, below $T_{3}$ improper ferroelectric. (After Ref. 13).

quence they have been frequently observed in displacive incommensurate phases. However, the phason branch has much lower frequency and is expected to be overdamped for small k; as a result it was not directly observed until recently. BWO spectroscopy succeeded for the first time to provide evidence for a phason mode in the incommensurate improper ferroelectric potassium selenite $\mathrm{K}_{2} \mathrm{SeO}_{4}$. The temperature dependence of dielectric losses in $\mathrm{K}_{2} \mathrm{SeO}_{4}$ at three frequencies is shown in Fig. 5. The incommensurate phase between $T_{3}$ and $T_{2}$ is characterized by an additional contribution to losses which can be fitted to a Debye relaxation with $(2 \pi \tau)^{-1} \cong 2 \mathrm{~cm}^{-1}$ near $T_{2}$ which softens down to $3 \times 10^{-3}$ $\mathrm{cm}^{-1}$ at $T_{3}$ as follows from subsequent microwave measurements by $\mathrm{M}$. Horioka et al. A group-theoretical analysis of selection rules in the incommensurate phase shows that the relaxation comes from a phason mode with a wave vector $\mathbf{q}$ equal to the wave vector $\mathbf{k}_{\mathrm{o}}$ of the incommensurate polarization wave. This result can be easily understood from Fig. 6 where the uniform $(q=0)$ dielectrically inactive and the $\mathbf{q}=\mathbf{k}_{\mathrm{o}}$ active phasons and amplitudons are schematically depicted. The decrease of the wave vector $\mathbf{k}_{\circ}$ as $T \rightarrow T_{3}$ causes an effective softening of the dielectric active phason mode and a consequent divergence of the corresponding $\varepsilon$ component.

\section{Conclusions}

As we have tried to illustrate, BWO spectroscopy is a technique for quantitative soft-mode studies in different types of ferroelectric. It enables one to obtain both the real and imaginary part of the dielectric response independently of any physical assumption or model. So far, however, very few BWO spectrometers are working properly so that dielectric data in the submillimetre and millimetre range are still rather scarce. We should like to stress that this spectral region is also of great interest for many other systems.

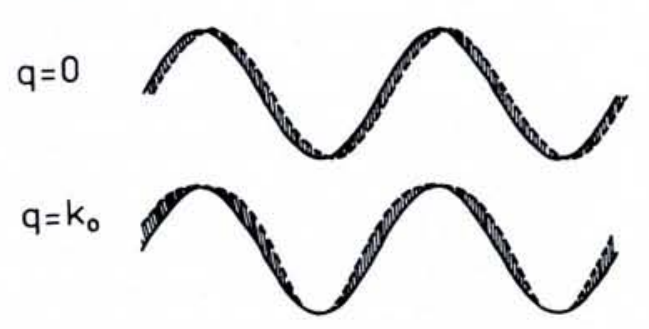

Fig. 6 - Long wavelength $\vec{q}=0$ and short wavelength $\vec{q}=\vec{k}_{o}$ phasons and amplitudons. The solid lines show the frozen-in polarization waves, the broken lines ana the hatched area show the excitations. Phason and amplitudon excitation are depicted on the left and right, respectively. Only the $\vec{q}=\vec{k}_{o}$ modes generate a net dipole moment and are therefore dielectrically active.

\section{Bibliography and References}

1. Bruce A.D. and Cowley R.A., Structural Phase Transitions (Taylor and Francis, London) 1981.

2. Murzin V.N., Pasynkov R.J. and Solo'ev S.P., Uspekhi Fiz. Nauk 92 (1967) 427. reelectrics and Antiferroelectrics (North Holland, Amsterdam) 1974.

4. Scott J.F., Rev. Mod. Phys. 46 (1974) 83. 5. Lines M.E. and Glass A.M., Principles and Applications of Ferroelectrics and Related Materials (Clarendon Press, Oxford) 1977.

6. Müller K.A., Luspin Y., Servoin J.L. and L-537.

7. Dvořák V., Ferroelectrics 7 (1974) 1.

8. Kozlov G.V., Volkov A.A., Scott J.F., Feldkamp G.E. and Petzelt J., Phys. Rev. B28 (1983) 255.

9. Volkov A.A., Kozlov G.V., Lebedev S.P. and Prokhorov A.M., Ferroelectrics 25 (1980) 531. P., Zh. Exp. Teor. Fiz. 79 (1980) 1430.

Europhysics News 13 (1982) 12.

12. Petzelt J., Phase Transitions 2 (1981) 155.

13. Petzelt J., Kozlov G.V., Volkov A.A. and shibashi Y., Z. Phys. B 33 (1979) 369.

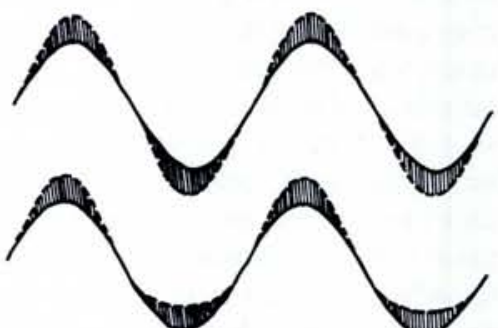

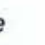

3. Blinc R. and Žekš B., Soft Modes in Fer-

\section{New Members of the European Physical Society}

\section{CATEGORY 4c)}

Danish Physical Society

K. Thomsen, Roskilde

\section{Eötvös Lorand Physical \\ Society \\ I. Kovacs, Budapest}

\section{French Physical Society}

F. Battistella, Toulouse

\section{German Physical Society \\ T. Dorfmüller, Bielefeld \\ H.J. Eichler, Berlin West \\ H.-U. Habermeier, Stuttgart \\ W. Hansen, Hamburg \\ H.J. Kluge, Mainz \\ B.F. Wortmann, Herne}

Hellenic Physical Society

J. Ventura, Iraklion
The Institute of Physics

S. Cox, Abingdon

T.J. Parker, London

Israel Physical Society

J. Katriel, Haifa

\section{The Netherlands' Physical Society}

P.P.J. van Engelen, Eindhoven

Norwegian Physical Society

K. Fredriksen, Alnabru

H.H. Grelland, Oslo

N.C. Hjorth, Oslo

Physical Section,

Union of Yugoslav Societies of Mathematicians,

Physicists and Astronomers

G. Bilalbegovic, Belgrade
Polish Physical Society

M.T. Czyzyk, Krakow

W. Duch, Torun

J. Janik, Krakow

W. Karas, Krakow

W. Minor, Warsaw

J. Mycielski, Warsaw

J. Pawelczyk, Warsaw

J. Pszczola, Krakow

P. Rusek, Wroclaw

J. Szwed, Krakow

Portuguese Physical Society

A.L.C. Anjo, Coimbra

J.M. Araújo, Porto

J. Carvalho Soares, Lisbon

M.A. Fortes, Lisbon

J.M. Machado da Silva, Porto

A.J.F. Praxedes, Lisbon

J.B. Sousa, Porto

Spanish Physical Society

B. Kasemo Mellerud, S 